\title{
Pharmacological Inhibition of NLRP3 Inflammasome Attenuates Myocardial Ischemia/Reperfusion Injury by Activation of RISK and Mitochondrial Pathways
}

\author{
Raffaella Mastrocola, ${ }^{1}$ Claudia Penna, ${ }^{1}$ Francesca Tullio, ${ }^{1}$ \\ Saveria Femminò, ${ }^{1}$ Debora Nigro, ${ }^{1}$ Fausto Chiazza, ${ }^{2}$ Loredana Serpe, ${ }^{2}$ \\ Debora Collotta, ${ }^{2}$ Giuseppe Alloatti, ${ }^{3}$ Mattia Cocco, ${ }^{2}$ \\ Massimo Bertinaria, ${ }^{2}$ Pasquale Pagliaro, ${ }^{1}$ Manuela Aragno, ${ }^{1}$ and Massimo Collino ${ }^{2}$ \\ ${ }^{1}$ Department of Clinical and Biological Sciences, University of Turin, Torino, Italy \\ ${ }^{2}$ Department of Drug Science and Technology, University of Turin, Torino, Italy \\ ${ }^{3}$ Department of Life Sciences and Systems Biology, University of Turin, Torino, Italy \\ Correspondence should be addressed to Massimo Collino; massimo.collino@unito.it
}

Received 7 September 2016; Revised 12 October 2016; Accepted 23 October 2016

Academic Editor: Cecilia Zazueta

\begin{abstract}
Copyright ( 2016 Raffaella Mastrocola et al. This is an open access article distributed under the Creative Commons Attribution License, which permits unrestricted use, distribution, and reproduction in any medium, provided the original work is properly cited.
\end{abstract}

\begin{abstract}
Although the nucleotide-binding oligomerization domain- (NOD-) like receptor pyrin domain containing 3 (NLRP3) inflammasome has been recently detected in the heart, its role in cardiac ischemia/reperfusion (IR) is still controversial. Here, we investigate whether a pharmacological modulation of NLRP3 inflammasome exerted protective effects in an ex vivo model of IR injury. Isolated hearts from male Wistar rats (5-6 months old) underwent ischemia (30 min) followed by reperfusion (20 or $60 \mathrm{~min})$ with and without pretreatment with the recently synthetized NLRP3 inflammasome inhibitor INF4E (50 $\mu \mathrm{M}, 20$ min before ischemia). INF4E exerted protection against myocardial IR, shown by a significant reduction in infarct size and lactate dehydrogenase release and improvement in postischemic left ventricular pressure. The formation of the NLRP3 inflammasome complex was induced by myocardial IR and attenuated by INF4E in a time-dependent way. Interestingly, the hearts of the INF4E-pretreated animals displayed a marked improvement of the protective RISK pathway and this effect was associated increase in expression of markers of mitochondrial oxidative phosphorylation. Our results demonstrate for the first time that INF4E protected against the IR-induced myocardial injury and dysfunction, by a mechanism that involves inhibition of the NLRP3 inflammasome, resulting in the activation of the prosurvival RISK pathway and improvement in mitochondrial function.
\end{abstract}

\section{Introduction}

Ischemic heart disease is one of the main culprits of illness and death $[1,2]$. The main outcome of a transient cardiac ischemia is the progressive decline of the left ventricle contractile function, frequently paralleled by impairment of the mitochondrial energy metabolism [3, 4]. During the reperfusion phase the sudden mitochondrial oxygen overload induces oxidative stress and further worsens the metabolic derangement [4], thus paradoxically exacerbating myocardial injury and inducing pyroptosis $[2,5]$. Pyroptosis is a caspase-1-dependent process leading to cell lysis, which has been demonstrated to be strongly regulated by the multiprotein platform complex nucleotide-binding oligomerization domain- (NOD-) like receptor pyrin domain containing 3 (NLRP3) inflammasome. The NLRP3 inflammasome comprises (a) NLRP3, (b) an apoptosis-associated speck-like protein containing a caspase activation recruitment domain (ASC), and (c) procaspase-1. In response to a wide range of danger signals, including oxygen-free radicals, $\mathrm{K}^{+}$efflux, or mitochondrial stress [6-8], NLRP3 recruits the adaptor protein ASC which in turn interacts with procaspase-1. 
Inflammasome oligomerization promotes the autocatalytic activation of procaspase- 1 and the processing of prointerleukin- (IL-) $1 \beta$ [2]. More recently, a new protein has been identified as member of the NLRP3 inflammasome complex, the Gasdermin D (GSDMD), which is recruited with kinetics similar to those required for caspase-1 activation. The proteolytic cleavage of GSDMD by caspase- 1 detaches its N-terminal fragment, which contributes to mediate IL$1 \beta$ secretion and pyroptosis [9]. Since NLRP3 is detectable in many cardiac cell types, including cardiofibroblasts (the most important cell type in the heart in terms of number of cells) and cardiomyocytes (the most important cell type in terms of cell volumes), it is likely that it may play a pivotal role in acute myocardial infarction $[10,11]$. Indeed, we and others have shown that NLRP3 is upregulated by ischemia/reperfusion (IR) injury and its myocardial activation is exacerbated by metabolic derangements $[12,13]$. Interestingly, genetic modulation of NLRP3 has been reported to reduce myocardial infarct sizes upon IR [13]. However, a very recent study failed to find any role of NLRP3 in determining myocardial IR injury [14] and another investigation supported cardioprotective effects due to NLRP3 inflammasome activation, thus highlighting that the interpretation of NLRP3 inflammasome role in myocardial IR injury is far from clear. Nevertheless, a cross-talk between NLRP3 and mitochondria, the main player of IR injury, has been described, with NLRP3 being able to sense the presence of reactive oxygen species (ROS) produced by normal or dysfunctional mitochondria [15]. Thus, the present study aimed to investigate the effects of a newly synthesized NLRP3 inflammasome inhibitor, named INF4E [16], in an ex vivo model of myocardial IR injury. We deepened our investigation evaluating its ability, in the rat heart, (i) to interfere with the IR-induced NLRP3 inflammasome activation and pyroptotic cascade and (ii) to improve the mitochondrial metabolic response to IR insult.

\section{Materials and Methods}

2.1. INF4E Preparation. INF4E was dissolved at $200 \mathrm{mM}$ concentration in DMSO. Stock solution was then diluted at a final concentration of $50 \mu \mathrm{M}$ in the perfusion buffer (see below). The description of the synthesis and the specificity of the inhibitor is included in the Supplemental Material (available online at http://dx.doi.org/10.1155/2016/5271251), according to previous publications $[16,17]$.

\subsection{Animals Protocol and Ex Vivo Ischemia/Reperfusion (IR)} Injury. Male Wistar rats (Harlan Laboratories, Udine, Italy) 5-6 months old, reaching a body weight of 450-550 g, were cared in compliance with the European Directive 2010/63/EU on the protection of animals used for scientific purposes. The animal protocols followed in this study were approved by the local "Animal Use and Care Committee." After one week of quarantine, with drink and food ad libitum, rats were anesthetized and killed. The hearts were rapidly perfused. A constant flow was maintained to obtain a typical coronary perfusion pressure of about $80 \mathrm{~mm} \mathrm{Hg}$ by the Langendorff technique with Krebs-Henseleit bicarbonate buffer containing
(mM) $\mathrm{NaCl} 118, \mathrm{NaHCO}_{3} 25, \mathrm{KCl} 4.7, \mathrm{KH}_{2} \mathrm{PO}_{4}$ 1.2, $\mathrm{MgSO}_{4}$ $1.2, \mathrm{CaCl}_{2} 1.25$, and Glucose 11. The buffer was gassed with $95 \% \mathrm{O}_{2}: 5 \% \mathrm{CO}_{2}$. The temperature of the perfusion system was maintained at $37^{\circ} \mathrm{C}$. The hearts underwent $30 \mathrm{~min}$ stabilization and then were exposed to $30 \mathrm{~min}$ of global no-flow, normothermic ischemia followed by a period of 20 or $60 \mathrm{~min}$ of reperfusion. Hearts from a subgroup of rats (IR+INF4E) were pretreated with $50 \mu \mathrm{M}$ INF4E in the perfusate for $20 \mathrm{~min}$ before ischemia (after the first 10 min of stabilization). Hearts from sham animals were exposed to 60 min perfusion only and served as reference group in Western blot analysis.

The hearts were electrically paced at $280-300 \mathrm{bpm}$ and kept in a temperature-controlled chamber $\left(37^{\circ} \mathrm{C}\right)$. Pacing was stopped at the beginning of the ischemia and restarted after the third min of reperfusion. Left ventricular pressure (LVP) and coronary perfusion pressure (CPP) were recorded and monitored with two electromanometers placed within the left ventricle and along the perfusion line, respectively. Coronary flow, CPP, and LVP were used as indices of preparation conditions. Moreover, end diastolic LVP was recorded as index of contracture development during I/R and developed ventricular pressure as index of contractile activity throughout the experiment using PowerLab data acquisition system and analyzed using Chart software (ADInstruments, Oxford, UK).

The perfusate flowing out of the heart was collected for measurement of lactate dehydrogenase (LDH) release $5 \mathrm{~min}$ immediately before ischemia and for the entire reperfusion period. To assess the conditions of experimental preparation the coronary flow rate was determined by the amount of perfusate measured in a specific time period. The heart was then cut in two parts by a coronal section (perpendicular to the long axis). The apical part (less than 1/3 of ventricular mass) was used for molecular analysis and, thus, frozen rapidly in liquid nitrogen and stored at $-80^{\circ} \mathrm{C}$. Infarct size assessment was performed by using the basal part of ventricle.

2.3. Measurement of the Infarct Size. Infarct mass was evaluated at the end of the reperfusion with the nitro-bluetetrazolium (NBT) technique by using a gravimetric method [18]. The basal part of the ventricles was dissected by transverse sections into two/ three slices, which were incubated for $20 \mathrm{~min}$ with a solution of NBT $(0.1 \%)$ in phosphate buffer. Two independent and blind observers carefully separated and then weighted both stained and unstained tissues. Total mass of necrosis was then calculated and expressed as percentage of ventricular mass. Since the ischemia was global and since we analyzed only the basal part of the ventricles the necrotic mass was expressed as a percentage of the analyzed ischemic tissue.

2.4. LDH Assay. Spectrophotometric analysis at $340 \mathrm{~nm}$ was performed on the collected perfusion effluent to measure $\mathrm{LDH}$ released from the heart.

2.5. Preparation of Tissue Extracts. Total proteins extracts were obtained from $10 \%(\mathrm{w} / \mathrm{v})$ apex homogenates in RIPA 
buffer $(0.5 \%$ Nonidet P- $40,0.5 \%$ sodium deoxycholate, $0.1 \%$ SDS, $10 \mathrm{mmol} / \mathrm{l} \mathrm{EDTA}$, and protease inhibitors) as previously described [19]. Protein concentrations were measured by Bradford assay (BioRad, Hercules, CA, USA) and samples were then stored at $-80^{\circ} \mathrm{C}$ for subsequent analysis.

2.6. Determination of IL-1 $\beta$ in Hearts Homogenates. Commercially available ELISA kit (R\&D Systems, Abingdon, UK) was used to measure concentrations of IL- $1 \beta$ in tissue homogenates, according to the manufacturer's instructions.

2.7. Western Blot Analysis. Total proteins extracts were separated by SDS-PAGE and blotted to nitrocellulose membrane (GE-Healthcare Europe, Milano, Italy). Membranes were incubated with rabbit anti-NLRP3 (Abcam, Cambridge, UK), rabbit anti-caspase-1 (Santa Cruz Biotechnology, Dallas, TX, USA), mouse anti-GSDMDC1 (Santa Cruz Biotechnology), rabbit anti-IL-1 $\beta$ (Santa Cruz Biotechnology), rabbit anti-caspase-1 (Santa Cruz Biotechnology, Dallas, TX, USA), mouse anti-Tyr ${ }^{204}$ ERK1/2 (Cell Signaling Technology), rabbit anti-total ERK1/2 (Cell Signaling Technology), mouse anti-Ser ${ }^{473}$ Akt (Cell Signaling Technology), rabbit anti-total Akt (Cell Signaling Technology), rabbit anti-Ser ${ }^{9}$ GSK-3 $\beta$ (Abcam, Cambridge, UK), anti-total GSK-3 $\beta$ (Cell Signaling Technology), rabbit antimitochondrial transcription factor A (mtTFA) (Novus Biologicals, Cambridge, UK), mouse anti-nuclear respiratory factor-1 (NRF-1) (Santa Cruz Biotechnology), and mouse anti-sarcomeric mitochondrial creatine kinase (sMtCK) (Santa Cruz Biotechnology) and then probed with proper HRP-conjugated secondary antibodies (BioRad). Clarity Western ECL substrate (BioRad) was used for protein detection and quantification was performed by densitometric analysis (Quantity-One, Bio-Rad software). Data were normalized according to the related antitubulin densitometric values.

2.8. Real-Time PCR. Total RNA was extracted from heart samples using the AllPrep ${ }^{\circledR}$ DNA/RNA/protein kit (Qiagen, Hilden, Germany), according to the manufacture instructions. The total RNA concentration $(\mu \mathrm{g} / \mathrm{mL})$ was determined by the fluorometer Qubit and the Quant-iT ${ }^{\mathrm{TM}}$ RNA Assay Kit (Invitrogen, Milano, Italy). A total of $500 \mathrm{ng}$ of RNA was reverse-transcribed using QuantiTect Reverse Transcription Kit (Qiagen). The synthesized cDNA was used for realtime polymerase chain reaction (RT-PCR). The cDNA was amplified by real-time PCR using SsoFast ${ }^{\mathrm{TM}}$ EvaGreen (Bio$\mathrm{Rad}$,) and primers specific for cytokine IL-1 $\beta$ (Mm_Illb_2_SG, cat. number QT01048355, Qiagen). The PCR reaction was performed at $95^{\circ} \mathrm{C}$ for $30 \mathrm{~s}$ followed by 40 cycles of $95^{\circ} \mathrm{C}$ for $5 \mathrm{~s}, 55^{\circ} \mathrm{C}$ for $10 \mathrm{~s}$. All samples were run in duplicate. At least two nontemplate controls were included in all PCR. The transcript of the reference gene ribosomal RNA 18S (Mm_Rn18s_3_SG, cat. number QT02448075, Qiagen) was used to normalize mRNA data, and the quantification data analyses were performed by using the Bio-Rad CFX Manager Software, version 1.6 (Bio-Rad) according to the manufacturer's instructions.
2.9. Materials. Compounds here used were obtained from the Sigma-Aldrich Company Ltd., unless otherwise stated.

2.10. Statistical Analysis. Data described in the text and figures are presented as means \pm standard error of the mean (s.e.m.) of $n$ observations, where $n$ represents the number of animals studied. Statistical analysis was performed using ANOVA test followed by Bonferroni's posttest. A $P$ value of less than 0.05 was considered to be statistically significant.

\section{Results}

3.1. INF4E Pretreatment Limits Infarct Size and Improves Contractility Recovery. Rat hearts exposed to a $30 \mathrm{~min}$ global ischemia and $60 \mathrm{~min}$ reperfusion developed a $60 \pm 3 \%$ infarct size in the basal portion of ventricle evaluated by NBT staining. When hearts were pretreated $(20 \mathrm{~min}$ prior to ischemia) with INF4E in the perfusate, the infarct size was significantly reduced compared to untreated hearts (IR) (Figure 1(a)). Importantly, LDH release in the perfusate was almost halved by drug treatment (Figure 1(b)). In addition, the INF4E pretreated hearts showed a twofold increase in contractile recovery after IR, as assessed by left ventricular pressure monitoring (Figure 1(c)).

Of note, IR caused an impairment of the mechanical performance, as evidenced by the dramatic reduction of developed LVP (DLVP) immediately after ischemia and the incomplete recovery during reperfusion. In fact, developed LVP fell from about $80 \mathrm{mmHg}$ in the preischemic condition to about $25 \mathrm{mmHg}$ after $10 \mathrm{~min}$ of reperfusion and then recovered to only $50 \mathrm{mmHg}$ at $60 \mathrm{~min}$ reperfusion, thus reaching only less than $65 \%$ of the preischemic value (Figures $1(c)$ and $1(d))$. The pretreatment with the NLRP3 inhibitor did not modify the recovery of DLVP at early reperfusion time (10 and $20 \mathrm{~min}$ ), whereas an improvement starts to be detectable after $40 \mathrm{~min}$, reaching values up to $85 \mathrm{mmHg}$ at $60 \mathrm{~min}$, corresponding to over $100 \%$ of the preischemic value at the end of reperfusion. The end diastolic LVP (EDLVP) was about $5 \mathrm{mmHg}$ during stabilization; then, the $30 \mathrm{~min}$ global ischemia and the subsequent reperfusion caused a sustained increase of this parameter in both the IR and IR+INF4E groups (Figure 1(e)). Actually, an increase of EDLVP was appreciated immediately after the end of ischemia and continued during the first $20 \mathrm{~min}$ of reperfusion. Then EDLVP further increased in the IR group reaching values over $30 \mathrm{~mm} \mathrm{Hg}$, while in the group pretreated with the NLRP3 inhibitor it decreased progressively to about $20 \mathrm{mmHg}$ after 1 hour of reperfusion. The increase in EDLVP was significantly $(P<0.05)$ attenuated by the NLRP3 inhibitor only at $60 \mathrm{~min}$ reperfusion.

3.2. INF4E Pretreatment Prevents NLRP3 Inflammasome Activation and Downstream Signaling. To confirm the ability of INF4E to interfere with NLRP3 inflammasome complex formation and activation in our experimental model, the expression level and the activation of the downstream signaling of NLRP3 inflammasome were assessed by Western blotting analysis in protein extracts obtained from the apical 


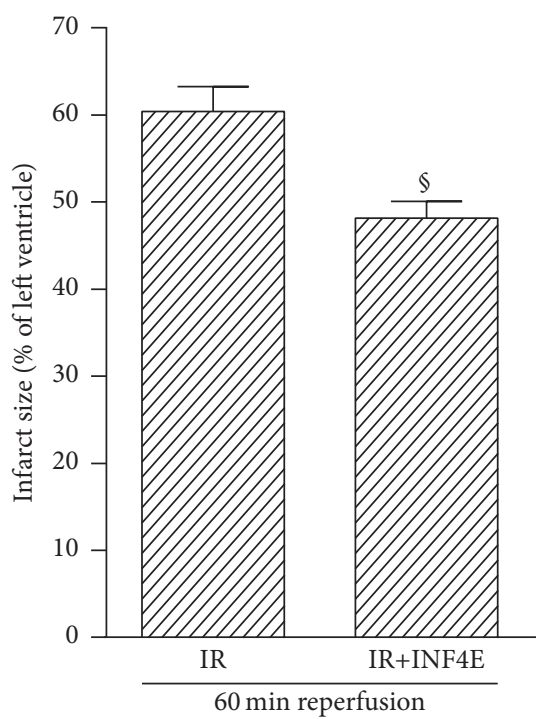

(a)

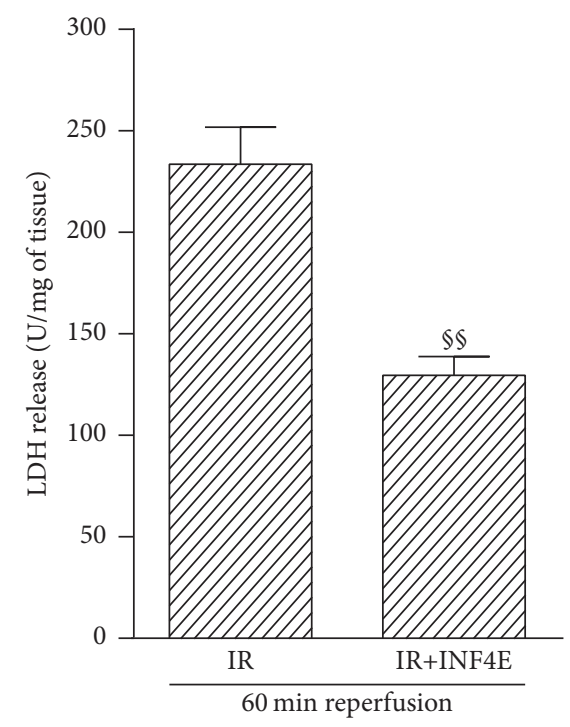

(b)

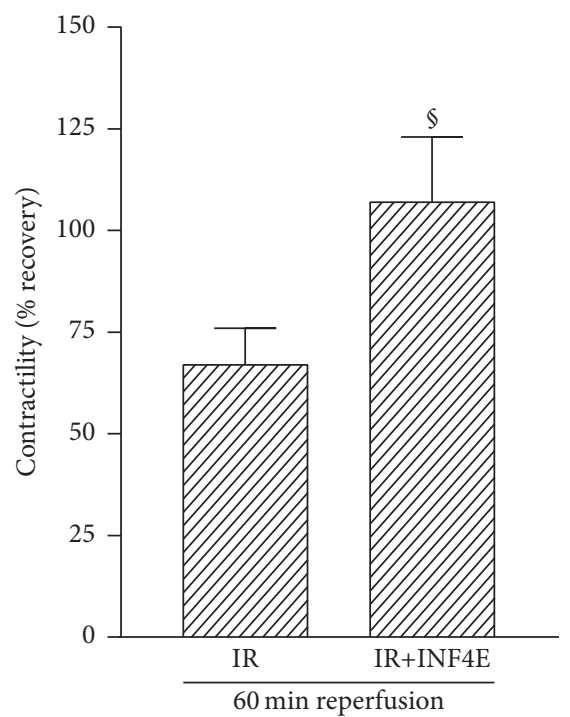

(c)

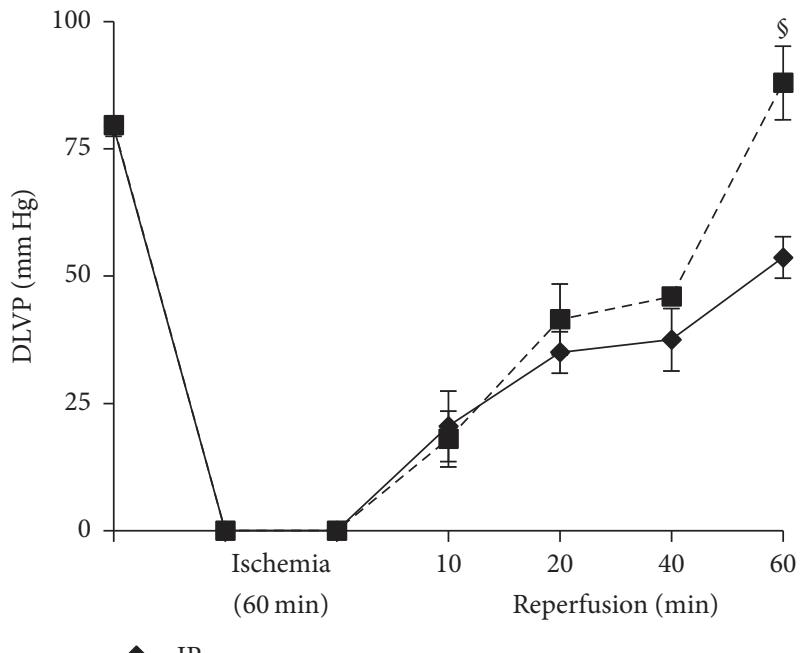

$\sim-$ IR
$-\square-$ IR+INF4E

(d)

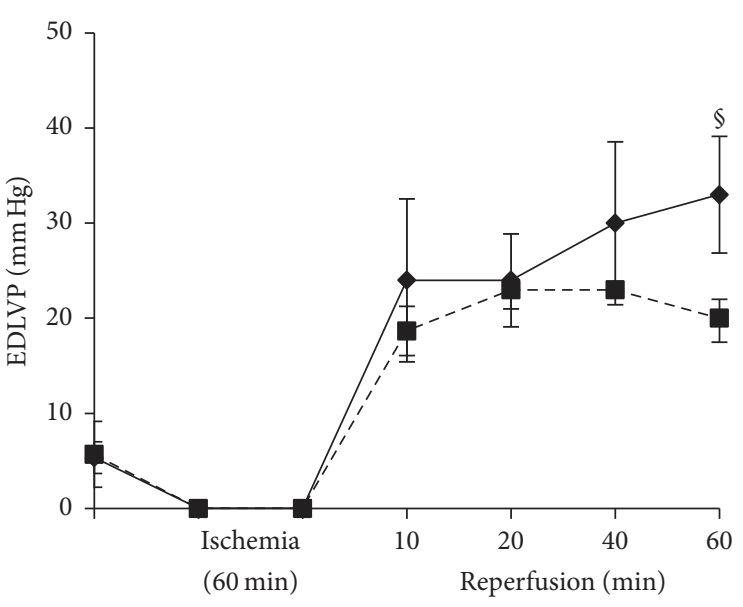

$\neg$ IR

IR+INF4E

(e)

FIGURE 1: Infarct area, LDH release, and contractility recovery in hearts from rats exposed to 30 min of ischemia plus 60 min of reperfusion, pretreated or not with $50 \mu \mathrm{M}$ INF4E in the perfusate $20 \mathrm{~min}$ before ischemia. (a): infarct size after IR exposition is expressed as a percentage of ischemic tissue (\% IS/IT). (b): LDH release in the perfusion effluent during the IR was expressed as units per mg of wet tissue weight. (c)-(e): monitoring of left ventricular pressure (LVP) was used to assess the contractility response to ischemia reperfusion injury. (c) shows the percentage of contractility recovered at the end of $60 \mathrm{~min}$ reperfusion. (d) and (e) show the entire time-course of developed LVP (DLVP) and end diastolic LVP (EDLVP), respectively. Data are means of 6 rats \pm SEM. ${ }^{\S} P<0.05$ versus IR. ${ }^{\$ \S} P<0.01$ versus IR.

portion of hearts pretreated or not with INF4E and exposed to IR (Figure 2). Besides, rat hearts were exposed to two different periods of reperfusion, short and long $(20 \mathrm{~min}$ and $60 \mathrm{~min}$, resp.) to better elucidate the kinetics of the pharmacological modulation of NLRP3 inflammasome and related pathways. As shown in Figure 2, the protein level of NLRP3 was increased in a time-dependent manner, reaching statistical significance only after the $60 \mathrm{~min}$ reperfusion. Notably, the INF4E pretreatment effectively reduced its upregulation. Western blotting analysis with an antibody recognizing the C-terminal region of caspase- 1 allowed the identification of two bands corresponding to the procaspase1 and the cleaved active p10 subunit of caspase-1. After $20 \mathrm{~min}$ of reperfusion the active form of caspase-1 was markedly increased, without significant changes in the expression of procaspase-1, although the INF4E-pretreated hearts showed a slight nonsignificant trend towards reduced procaspase cleavage. After $60 \mathrm{~min}$ of reperfusion, both precursor and active forms of caspase-1 were dramatically increased in the untreated IR group, whereas the INF4E-pretreatment significantly prevented procaspase cleavage. As shown in Figure 2(c), caspase-1 activation is associated with cleavage 

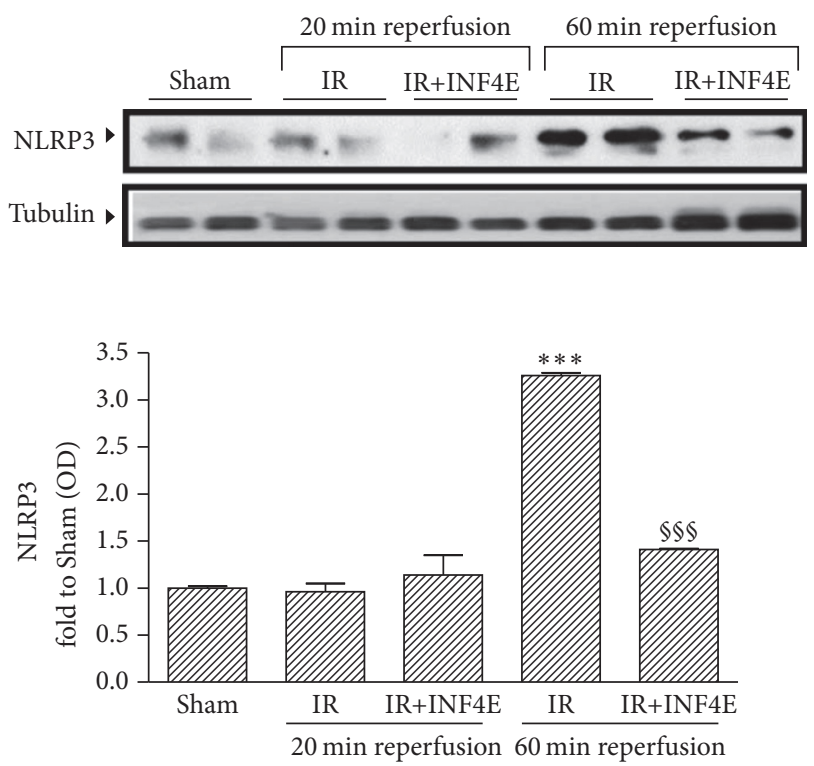

(a)
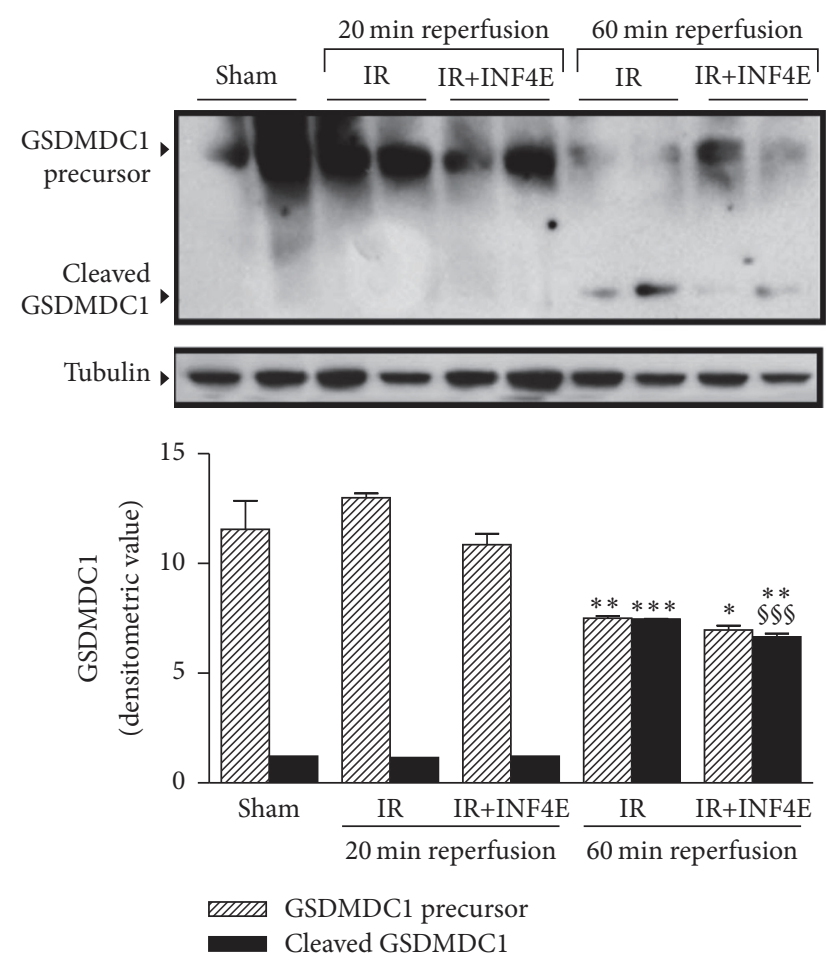

(c)

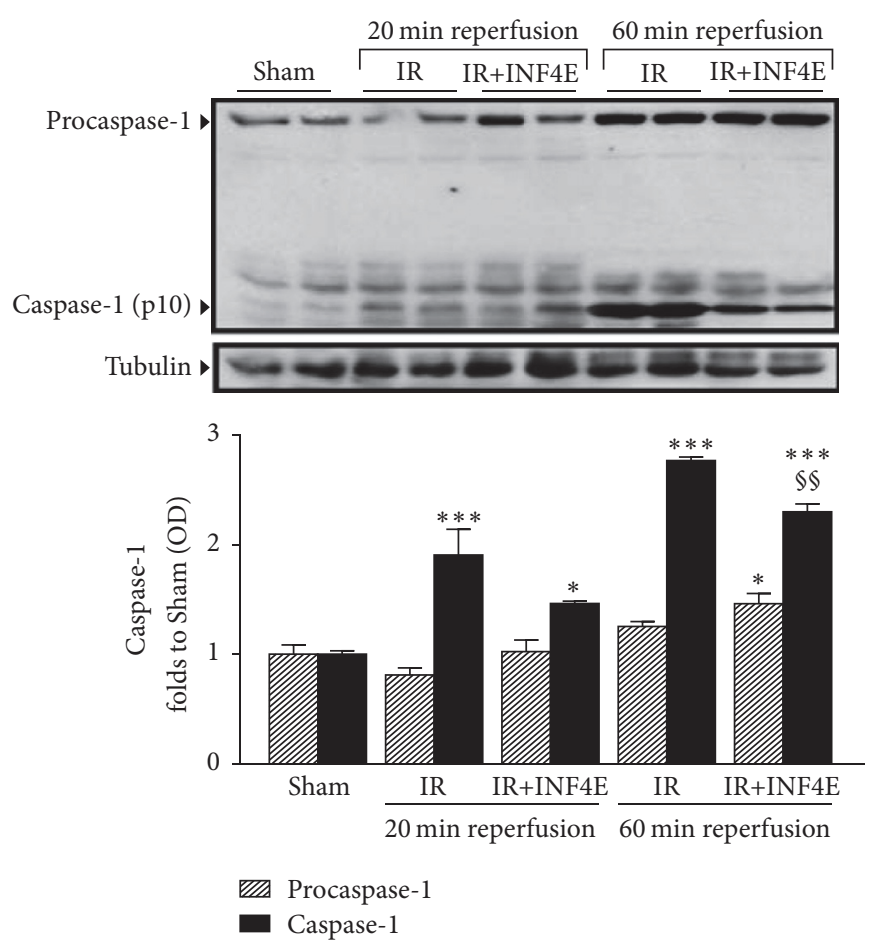

(b)
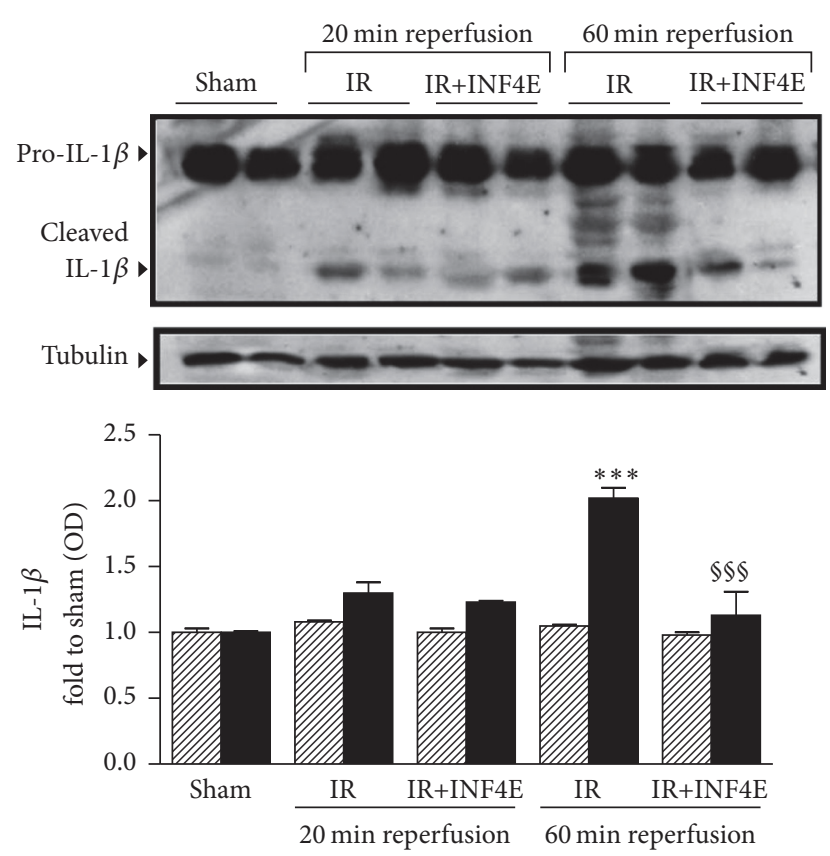

VIIII Pro-IL-1 $\beta$

Cleaved IL-1 $\beta$

(d)

FIGURE 2: Inflammasome expression and activation in the rat heart exposed to $30 \mathrm{~min}$ ischemia plus 20 or 60 min reperfusion, pretreated or not with INF4E. Representative Western blotting showing cardiac levels of NLRP3 and of downstream activation of caspase-1, GSDMDC1, and IL-1 $\beta$ cleavage assessed on heart extracts. Histograms report densitometric analysis normalized for the corresponding tubulin content. Data are means of 6 rats \pm SEM. ${ }^{*} P<0.05,{ }^{* *} P<0.01$, and ${ }^{* * *} P<0.001$ versus Sham ${ }^{\$ \S} P<0.01,{ }^{\$ \S \$} P<0.001$ versus IR. 


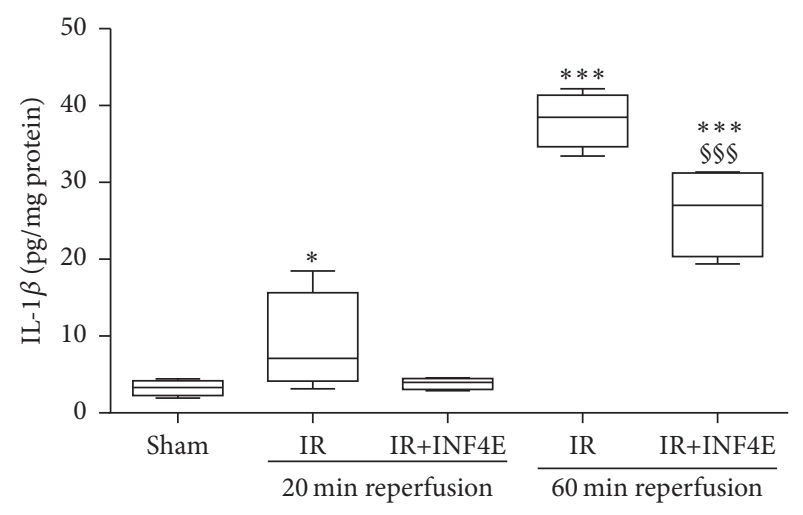

(a)

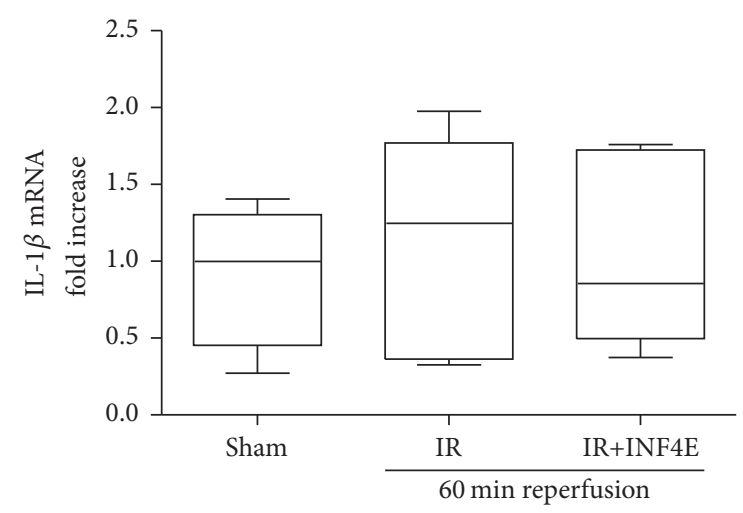

(b)

FIGURE 3: IL-1 $\beta$ concentrations evaluated by ELISA (a) and mRNA levels of IL-1 $\beta$ measured by RT-PCR (b) in extracts of rat hearts exposed to $30 \mathrm{~min}$ ischemia plus 20 or 60 min reperfusion, pretreated or not with INF4E. Data are means of 6 rats \pm SEM. ${ }^{*} P<0.05$, ${ }^{* * *} P<0.001$ versus Sham; ${ }^{\S \S \$} P<0.001$ versus IR.

of the GSDMDCl component of the inflammasome platform, detectable only after the longest reperfusion time. Interestingly, INF4E pretreatment significantly reduced GSDMCD1 cleavage. Accordingly, the protein levels of the active form of IL-1 $\beta$ showed a robust increase after the $60 \mathrm{~min}$ reperfusion, as demonstrated by Western blot analysis (Figure 2) and confirmed by ELISA (Figure 3(a)). The INF4E pretreatment prevented the slight increase in IL- $1 \beta$ production due to $20 \mathrm{~min}$ of reperfusion and strongly reduced the massive IL-1 $\beta$ release recorded after $60 \mathrm{~min}$ of reperfusion (Figures 2 and 3(a)). Interestingly, neither the IR injury nor the drug treatment affected the mRNA levels of IL-1 $\beta$ (Figure 3(b)), thus confirming a selective effect of INF4E on NLRP3 inflammasome-dependent IL-1 $\beta$ cleavage rather than expression.

\subsection{INF4E Pretreatment Enhances RISK Pathway Protective} Activity. Very recently, a role for NLRP3 inflammasome activation in the modulation of the Reperfusion Injury Salvage Kinase (RISK) pathway has been suggested [20]. Thus, we quantified expression and activity (in terms of phosphorylation) of the key members of this pathway. After 20 min of reperfusion no modulation of the activity and/or expression level of members of the RISK pathway was recorded (Figure 4). On the contrary, the longer IR challenging induced increased phosphorylation rate of both ERK1/2 (Figures 4(a) and 4(b)) and GSK-3 $\beta$ (Figures $4(\mathrm{a})$ and $4(\mathrm{~d})$ ), while phosphorylation of Akt tended to increase, without reaching statistical significance, in untreated hearts exposed to either short or long IR protocol (Figures 4(a) and 4(c)). Interestingly, the INF4E pretreatment further increased the phosphorylation rate of ERK1/2, Akt, and GSK-3 $\beta$ induced by the $60 \mathrm{~min}$ reperfusion in untreated hearts (Figures $4(\mathrm{~b})$ and $4(\mathrm{c})$ ), suggesting an enhancement of the activation of this protective pathway by the pharmacological intervention. and Energy Metabolism. Since mitochondrial metabolism is highly involved in the myocytes response to ischemic insult, and a cross-talk between mitochondria and NLRP3 has been described [21], we analyzed markers of mitochondrial biogenesis after $30 \mathrm{~min}$ ischemia and $60 \mathrm{~min}$ reperfusion. The MtTFA and the NRF-1 were markedly downregulated by IR. Conversely, mitogenesis was significantly preserved by INF4E pretreatment (Figures 5(a)-5(c)). A crucial physiological reaction of cardiac myocytes to oxygen deprivation is the enhancement of mitochondrial energy production, as suggested in our model by the increased expression of sMtCK after IR (Figures 5(a) and 5(d)). Intriguingly, the INF4E pretreatment further stimulated the expression of sMtCK by $25 \%$ with respect to untreated rat hearts (Figures 5(a) and $5(d))$.

\section{Discussion}

The present study improves our understanding on the effects of the NLRP3 inflammasome targeting in acute myocardial infarction. Here we confirmed that myocardial IR induces transcription of all the inflammasome components in a timedependent way, with slight effect detectable when hearts were exposed to $20 \mathrm{~min}$ reperfusion and robust overexpression and activation at the longest reperfusion time $(60 \mathrm{~min})$. These data are in agreement with previous papers showing increased expression levels of NLRP 3 and procaspase- 1 in the infarcted and noninfarcted areas in both cardiomyocytes and nonmyocyte cell, associated with augmentation of caspase1 activity [13,22]. We also previously demonstrated that an enhanced susceptibility to a myocardial ischemic insult, due to metabolic derangements, is paralleled by greater NLRP3 inflammasome activation in the heart [12]. However, the potential role of the innate immune NLRP3 protein complex as therapeutic target for cardiac infarction is ill defined. This is mainly due to the contrasting results so far obtained with targeted deletion of the inflammasome components. Few studies demonstrated that the depletion of even one of the inflammasome complex components, either the sensor (NLRP3) or the effector enzyme (caspase-1), can prevent its activation 

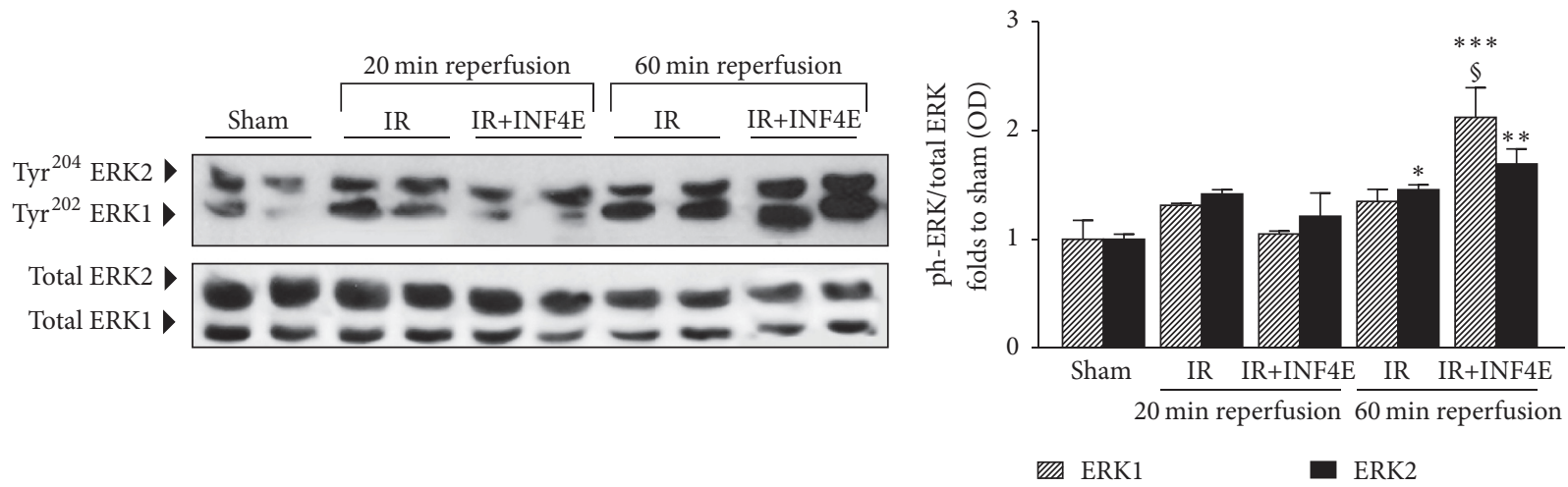

(b)
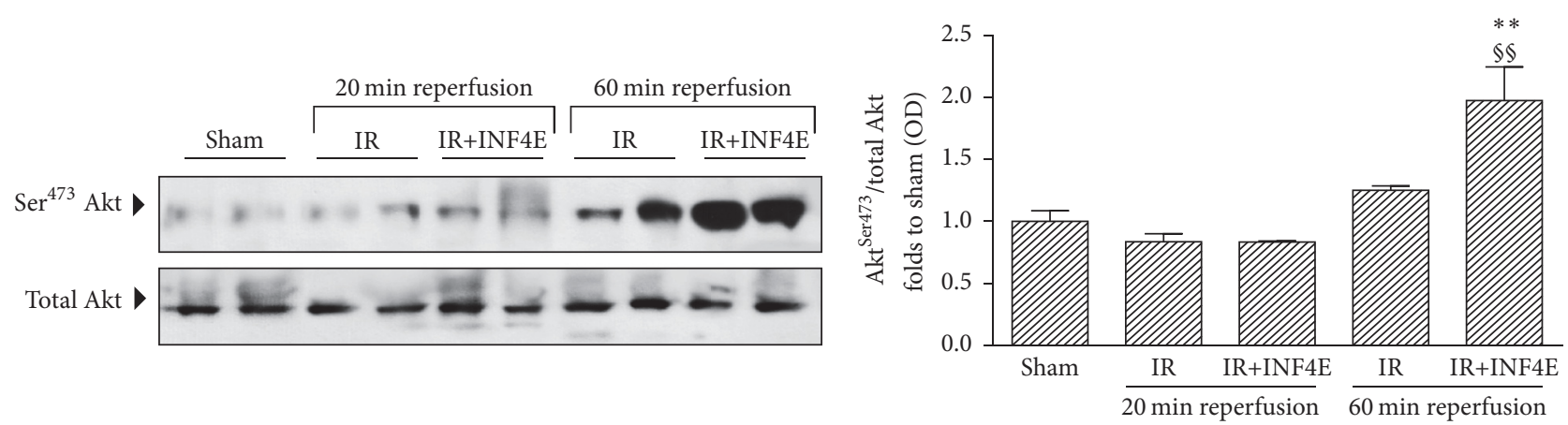

(c)

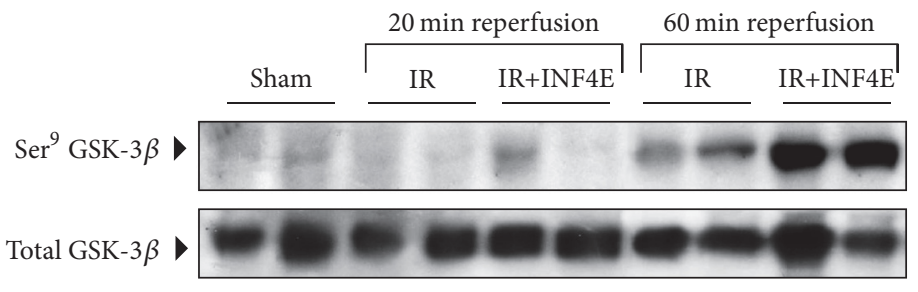

(a)

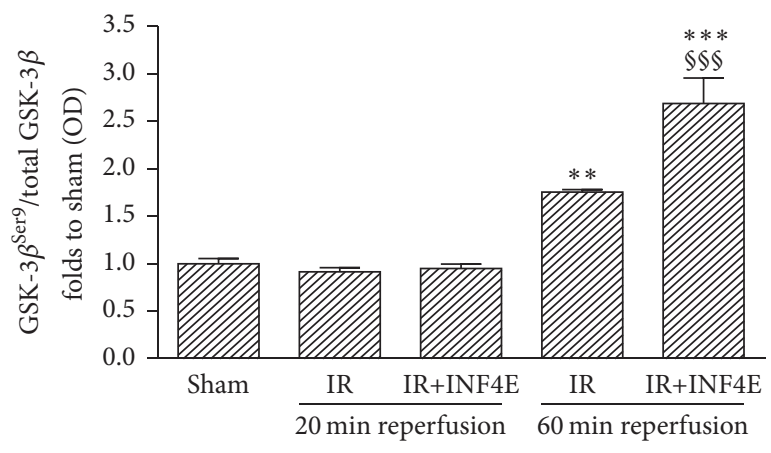

(d)

FIgURE 4: Prosurvival RISK pathway activation in the rat hearts exposed to $30 \mathrm{~min}$ ischemia plus 20 or 60 min reperfusion, pretreated or not with INF4E. (a) Representative Western blotting for cardiac levels of total ERK1/2 expression and Thr202/Tyr204 and Thr185/Tyr187 phosphorylation, respectively, total Akt protein expression and Ser473 phosphorylation, and total GSK-3 $\beta$ protein expression and Ser9 phosphorylation, performed on heart extracts. (b)-(d) Histograms report densitometric analysis of the phosphorylated-to-total form ratio. Data are means of 6 rats \pm SEM. ${ }^{*} P<0.05,{ }^{* *} P<0.005$, and ${ }^{* * *} P<0.001$ versus Sham; ${ }^{\S} P<0.05,{ }^{\S \S} P<0.005$, and ${ }^{\S \S \S} P<0.001$ versus IR.

and protect the heart preventing ischemic injury and adverse cardiac remodelling [22-24]. In contrast, Sandanger et al. [20] showed that absence of NLRP3 results in increased myocardial infarct size after in vivo IR, whereas Jong et al. [14] concluded that NLRP3 plays no role in acute myocardial infarction due to low cardiac expression. As stated by the same authors, significant differences in the used methodologies as well as in the specific endpoint of interest may help to explain this inconsistency [25]. Besides, a discrepancy in the outcome between $\mathrm{NLRP}^{-/-}$and $\mathrm{ASC}^{-/-}$mice after cardiac IR has been previously observed [13, 26], suggesting the existence of important inflammasome-independent effects related to targeted genetic deficiency. Thus, only the evaluation of small molecules able to selectively inhibit the NLRP3 inflammasome may allow ultimate elucidation of the potential of NLRP3 inflammasome as pharmacological target for therapeutic intervention in myocardial IR injury. Unfortunately, the lack of highly selective pharmacological inhibitors limits the investigation. INF4E is one of the few compounds that has been demonstrated to directly target 

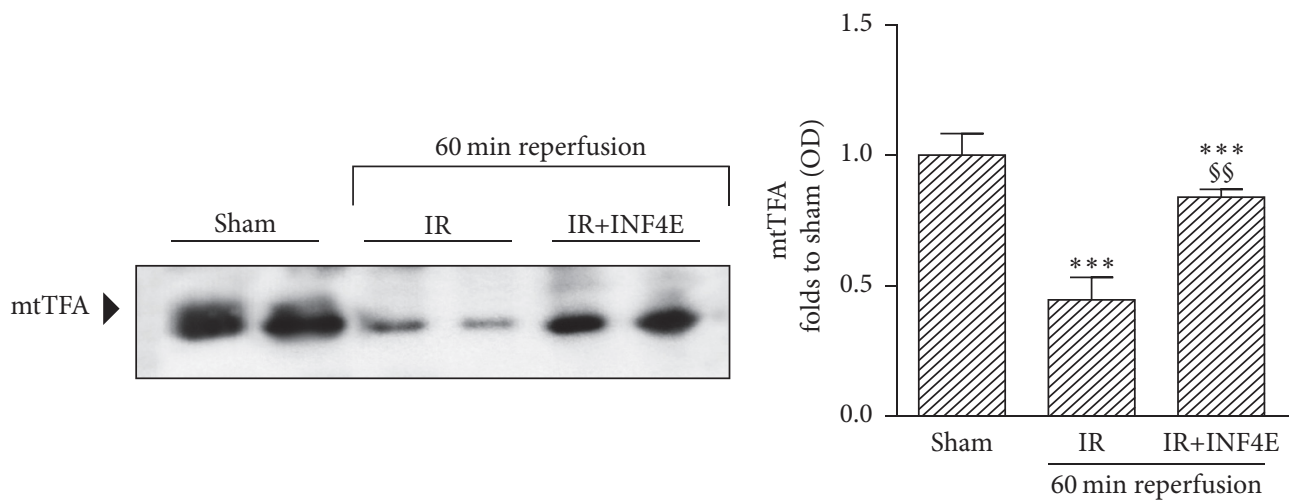

(b)

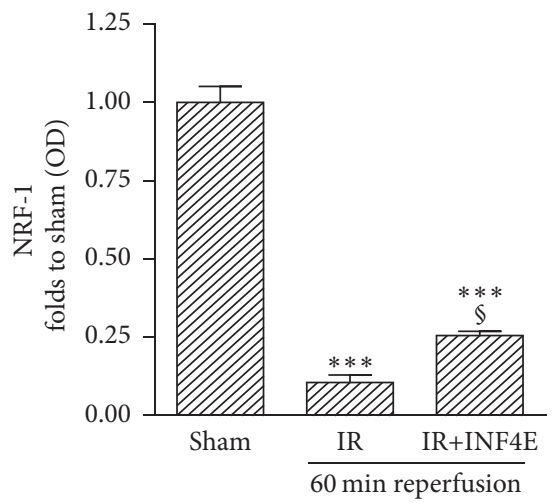

(c)

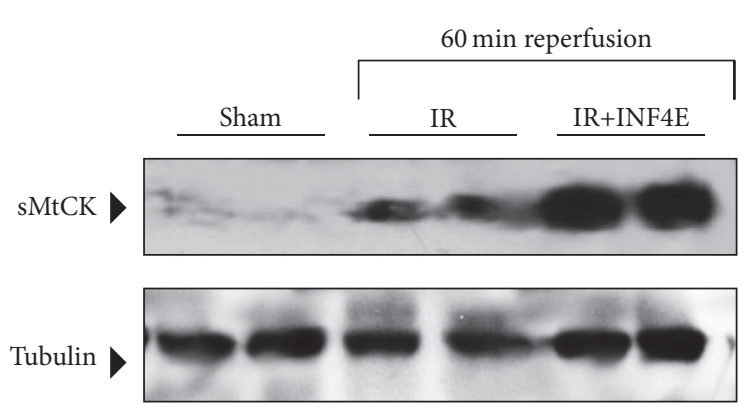

(a)

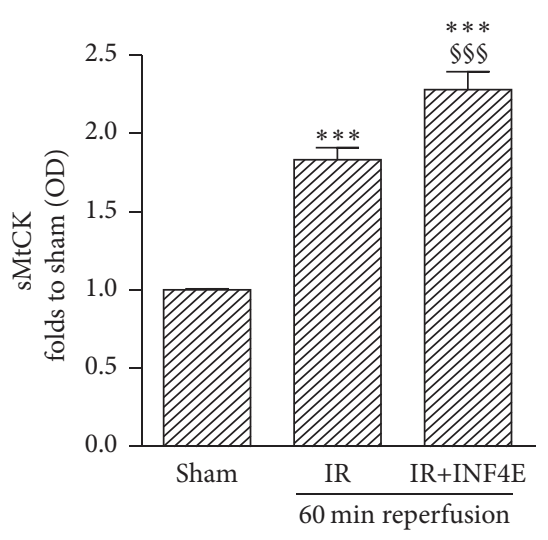

(d)

FIGURE 5: Mitochondrial biogenesis and energy metabolism evaluated in the rat hearts exposed to 30 min ischemia plus 20 or 60 min reperfusion, pretreated or not with INF4E. (a) Representative Western blotting for cardiac levels of MtTFA, NRF-1, and sMtCK performed on heart extracts. (b)-(d) Histograms report densitometric analysis normalized for the corresponding tubulin content. Data are means of 6 rats \pm SEM. ${ }^{* * *} P<0.001$ versus Sham; ${ }^{\S} P<0.05,{ }^{\$ \S} P<0.005$, and ${ }^{\$ \S \$} P<0.001$ versus IR.

the NLRP3 inflammasome and inhibit the ATPase activity of NLRP3 required for its activation. Although there are clear indications that this drug exerts a specific effect on NLRP3 inflammasome independently of the activating stimulus, the exact mechanism of action has still to be clarified [16]. Here we demonstrate, for the first time, that administration of the NLRP3 inflammasome inhibitor INF4E in a single dose significantly reduces infarct size, the main endpoint to target in cardioprotective studies. Moreover, the pretreatment with INF4E preserves systolic function, an index of reduced myocardial stunning. To the best of our knowledge, so far, only another small molecule acting as NLRP3 inflammasome inhibitor has been tested in models of acute myocardial injury, showing protective effects similar to those recorded in our ex vivo model of myocardial IR injury [27, 28]. Interestingly, this compound did not reduce infarct size at 
$3 \mathrm{~h}$ of reperfusion, while it significantly reduced infarct size at $24 \mathrm{~h}$, if administered at the beginning of reperfusion, but not after $3 \mathrm{~h}$ reperfusion [29], thus confirming the importance of the first period of reperfusion for IR injury development and the efficacy of pharmacological strategies [5]. Here we documented significant reduction of infarct size even after $60 \mathrm{~min}$ of reperfusion, with INF4E administered as pretreatment. It has to be noted that our ex vivo model of IR injury may allow excluding the involvement of infiltrating inflammatory cells that usually may affect the reperfusion-related injury as documented in the previous studies investigating the effects of NLRP3 inhibitors when given during reperfusion $[28,29]$. Thus, our data further extend the previous findings suggesting that the continuous pharmacological inhibition of NLRP3 inflammasome pathway already during the ischemic period may significantly contribute to the beneficial effects recorded at the end of reperfusion. The kinetics of the reperfusion injury may also affect the entity of the drug target expression and, thus, protection. In fact, when comparing the $20 \mathrm{~min}$ reperfusion and the $60 \mathrm{~min}$ reperfusion models we observed a progressive expression and activity of NLRP3 inflammasome complex, thus confirming previous findings on the timing of NLRP3 inflammasome formation in the heart during ischemia reperfusion [29].

Very recently, a role for NLRP3 inflammasome activation in the cardioprotective RISK pathway has been faintly suggested, but not convincingly demonstrated [20]. Here we measured the entire RISK pathway, which includes Akt and ERK1/2 activation and GSK3 $\beta$ inhibition through phosphorylation [30]. Our data demonstrate that the activation of ERK/Akt/GSK-3 $\beta$ signaling is further enhanced by pharmacological inhibition of the NLRP 3 inflammasome complex and this effect may significantly contribute to its cardioprotective effects. These data confirm our previous findings, showing that pharmacological inhibition of NLRP3 inflammasome significantly potentiates the activity of the prosurvival Akt pathway [31]. Besides, the cross-talk between NLRP3 inflammasome and RISK pathways is further confirmed by the comparative analysis of the expression/activity of members of both pathways, which show robust increase only in the $60 \mathrm{~min}$ reperfusion model in both cases.

Although we demonstrated a direct effect of the tested compound on NLRP3 inflammasome activation, we cannot rule out the potential interactions of INF-4E with other signaling pathways, including those involved in the regulation of the expression of NLRP3 and/or activation of the RISK pathway. Thus, a further rigorous evaluation of effects of the tested compound on other signaling pathways affected by IR is needed to better elucidate its pharmacodynamics profile.

The effects of the pharmacological inhibition of NLRP3 inflammasome on the RISK pathway are closely related to those recorded on mitochondrial metabolic response. Indeed, the mitochondrial metabolic dysfunction is crucial for cardiac damage development, as both the block of mitochondrial ATP-sensitive potassium channel (mitoKATP) and the opening of the mitochondrial permeability transition pore (mPTP) are common end effectors of the IR injury. Accordingly, pharmacological strategies which aimed to open the mitoKATP and inhibit $\mathrm{mPTP}$ opening effectively reduce myocardial IR injury and improve cardiomyocytes energy homeostasis via modulation of the activity of members of the RISK pathway [32-35]. We and others have demonstrated that the Akt-mediated inactivation of GSK-3 $\beta$ is critical for the prevention of myocardial IR injury, being a key event in the regulation of apoptosis and the enhancement of mitochondrial biogenesis [36-38]. Besides, the use of selective GSK-3 $\beta$ inhibitors evokes protection against IR injury and promotes cell survival by limiting $\mathrm{MPTP}$ opening $[39,40]$. In agreement with the above-mentioned observations, the INF4E-induced amplification of the RISK pathway activation here described positively impacted on mitochondrial metabolism. In fact, our data clearly demonstrate that markers of mitochondrial biogenesis, suppressed by IR, were significantly upregulated by INF4E administration. Besides, the administered compound enhanced mitochondrial energy metabolism, shown in terms of reinforced expression of the sMtCK, which is a marker of increased mitochondrial ATP production.

Unfortunately, our study does not allow identifying the specific cell types involved in NLRP3-mediated responses. Cardiomyocytes are the most prominent cell type in the heart and loss of contractile tissue is the most important consequence of a myocardial infarction. However, in cardiomyocytes, the activation on NLRP3 inflammasome evokes caspase- 1 activation and pyroptosis, but not relevant release of mature IL- $1 \beta[22,41]$. In contrast, NLRP3 inflammasome activation in myocardial fibroblasts induces the production of large amounts of mature IL- $1 \beta$, causing a rapid amplification of the inflammatory response [13, 23, 42]. As cardiomyocytes are crucial target for the IL- $1 \beta$ produced by resident fibroblasts, which impairs contractile function and induces apoptotic cell death, we may speculate that the beneficial effects of INF4E are due, at least in part, to an indirect improvement in cardiomyocytes functionality.

\section{Conclusion}

Taken together, our results strengthen the crucial role of NLRP3 inflammasome activation even in the early step of myocardial injury caused by IR and show, for the first time, that its pharmacological inhibition by INF4E reduced the organ injury/dysfunction. Preservation of cardiac function by INF4E is, at least in part, attributable to a cardioprotective effect mediated by the activation of the RISK survival pathways and the improvement in mitochondrial function, which, in turn, may improve cardiac function and postischemic outcome. It has to be stressed, however, that the lack of long-term evaluation as well as data on drug effects when administered during reperfusion limit the interpretation of the clinical transferability of our findings. Thus, further rigorous evaluations are needed to gain a better understanding of both efficacy and the mechanism of action of INF4E in the settings of acute myocardial infarction.

\section{Competing Interests}

The authors declare that they have no competing interests. 


\section{Acknowledgments}

This work was supported and funded by the University of Turin (Ricerca Locale 2015 Linea A and Linea B).

\section{References}

[1] A. S. Go, D. Mozaffarian, V. L. Roger et al., "Executive summary: heart disease and stroke statistics-2013 update: a report from the American Heart Association," Circulation, vol. 127, no. 1, pp. 143-152, 2013.

[2] M. Takahashi, "NLRP3 inflammasome as a novel player in myocardial infarction," International Heart Journal, vol. 55, no. 2, pp. 101-105, 2014.

[3] M. G. Perrelli, P. Pagliaro, and C. Penna, "Ischemia/reperfusion injury and cardioprotective mechanisms: role of mitochondria and reactive oxygen species," World Journal of Cardiology, vol. 3, no. 6, pp. 186-200, 2011.

[4] C. Penna, M.-G. Perrelli, and P. Pagliaro, "Mitochondrial pathways, permeability transition pore, and redox signaling in cardioprotection: therapeutic implications," Antioxidants \& Redox Signaling, vol. 18, no. 5, pp. 556-599, 2013.

[5] D. M. Yellon and D. J. Hausenloy, "Myocardial reperfusion injury," The New England Journal of Medicine, vol. 357, no. 11, pp. 1121-1135, 2007.

[6] R. Zhou, A. Tardivel, B. Thorens, I. Choi, and J. Tschopp, "Thioredoxin-interacting protein links oxidative stress to inflammasome activation," Nature Immunology, vol. 11, no. 2, pp. 136-140, 2010.

[7] R. Zhou, A. S. Yazdi, P. Menu, and J. Tschopp, "A role for mitochondria in NLRP3 inflammasome activation," Nature, vol. 469, no. 7329, pp. 221-225, 2011.

[8] J. R. Yaron, S. Gangaraju, M. Y. Rao et al., "K+ regulates Ca2+ to drive inflammasome signaling: dynamic visualization of ion flux in live cells," Cell Death and Disease, vol. 6, no. 10, Article ID e1954, 2015.

[9] X. Liu, Z. Zhang, J. Ruan et al., "Inflammasome-activated gasdermin D causes pyroptosis by forming membrane pores," Nature, vol. 535, no. 7610, pp. 153-158, 2016.

[10] J. A. Kummer, R. Broekhuizen, H. Everett et al., "Inflammasome components NALP 1 and 3 show distinct but separate expression profiles in human tissues suggesting a site-specific role in the inflammatory response," Journal of Histochemistry and Cytochemistry, vol. 55, no. 5, pp. 443-452, 2007.

[11] N. A. Bracey, P. L. Beck, D. A. Muruve et al., “The Nlrp3 inflammasome promotes myocardial dysfunction in structural cardiomyopathy through interleukin-1 $\beta$," Experimental Physiology, vol. 98, no. 2, pp. 462-472, 2013.

[12] R. Mastrocola, M. Collino, C. Penna et al., "Maladaptive modulations of nlrp3 inflammasome and cardioprotective pathways are involved in diet-induced exacerbation of myocardial ischemia/reperfusion injury in mice," Oxidative Medicine and Cellular Longevity, vol. 2016, Article ID 3480637, 12 pages, 2016.

[13] Ø. Sandanger, T. Ranheim, L. E. Vinge et al., "The NLRP3 inflammasome is up-regulated in cardiac fibroblasts and mediates myocardial ischaemia-reperfusion injury," Cardiovascular Research, vol. 99, no. 1, pp. 164-174, 2013.

[14] W. M. Jong and C. J. Zuurbier, "A role for NLRP3 inflammasome in acute myocardial ischaemia-reperfusion injury?" Cardiovascular Research, vol. 99, no. 1, p. 226, 2013.
[15] J. Zhou and W.-J. Chng, "Roles of thioredoxin binding protein (TXNIP) in oxidative stress, apoptosis and cancer," Mitochondrion, vol. 13, no. 3, pp. 163-169, 2013.

[16] M. Cocco, D. Garella, A. Di Stilo et al., "Electrophilic warheadbased design of compounds preventing NLRP3 inflammasomedependent pyroptosis," Journal of Medicinal Chemistry, vol. 57, no. 24, pp. 10366-10382, 2014.

[17] M. Cocco, G. Miglio, M. Giorgis et al., "Design, synthesis, and evaluation of acrylamide derivatives as direct NLRP3 inflammasome inhibitors," ChemMedChem, vol. 11, no. 16, pp. 17901803, 2016.

[18] C. Penna, M. Brancaccio, F. Tullio et al., "Overexpression of the muscle-specific protein, melusin, protects from cardiac ischemia/reperfusion injury," Basic Research in Cardiology, vol. 109, no. 4, article 418, 2014.

[19] M. Aragno, R. Mastrocola, C. Ghé et al., "Obestatin induced recovery of myocardial dysfunction in type 1 diabetic rats: underlying mechanisms," Cardiovascular Diabetology, vol. 11, article 129, 2012.

[20] Ø. Sandanger, E. Gao, T. Ranheim et al., "NLRP3 inflammasome activation during myocardial ischemia reperfusion is cardioprotective," Biochemical and Biophysical Research Communications, vol. 469, no. 4, pp. 1012-1020, 2016.

[21] P. Gurung, J. R. Lukens, and T. D. Kanneganti, "Mitochondria: diversity in the regulation of the NLRP3 inflammasome," Trends in Molecular Medicine, vol. 21, no. 3, pp. 193-201, 2015.

[22] E. Mezzaroma, S. Toldo, D. Farkas et al., "The inflammasome promotes adverse cardiac remodeling following acute myocardial infarction in the mouse," Proceedings of the National Academy of Sciences of the United States of America, vol. 108, no. 49, pp. 19725-19730, 2011.

[23] M. Kawaguchi, M. Takahashi, T. Hata et al., "Inflammasome activation of cardiac fibroblasts is essential for myocardial ischemia/reperfusion injury," Circulation, vol. 123, no. 6, pp. 594-604, 2011.

[24] Y. Liu, K. Lian, L. Zhang et al., “TXNIP mediates NLRP3 inflammasome activation in cardiac microvascular endothelial cells as a novel mechanism in myocardial ischemia/reperfusion injury," Basic Research in Cardiology, vol. 109, no. 5, article 415, 2014.

[25] S. Toldo, C. Marchetti, and A. Abbate, "nLRP3 inflammasome activation during myocardial ischemia reperfusion is cardioprotective," Biochemical and Biophysical Research Communications, vol. 470, no. 4, pp. 811-812, 2016.

[26] C. J. Zuurbier, W. M. C. Jong, O. Eerbeek et al., "Deletion of the innate immune NLRP3 receptor abolishes cardiac ischemic preconditioning and is associated with decreased IL-6/STAT3 signaling," PLoS ONE, vol. 7, no. 7, Article ID e40643, 2012.

[27] C. Marchetti, J. Chojnacki, S. Toldo et al., "A novel pharmacologic inhibitor of the NLRP3 inflammasome limits myocardial injury after ischemia-reperfusion in the mouse," Journal of Cardiovascular Pharmacology, vol. 63, no. 4, pp. 316-322, 2014.

[28] C. Marchetti, S. Toldo, J. Chojnacki et al., "Pharmacologic inhibition of the NLRP3 inflammasome preserves cardiac function after ischemic and nonischemic injury in the mouse," Journal of Cardiovascular Pharmacology, vol. 66, no. 1, pp. 1-8, 2015.

[29] S. Toldo, C. Marchetti, A. G. Mauro et al., "Inhibition of the NLRP3 inflammasome limits the inflammatory injury following myocardial ischemia-reperfusion in the mouse," International Journal of Cardiology, vol. 209, pp. 215-220, 2016.

[30] D. J. Hausenloy, S. Lecour, and D. M. Yellon, "Reperfusion injury salvage kinase and survivor activating factor enhancement 
prosurvival signaling pathways in ischemic postconditioning: two sides of the same coin," Antioxidants and Redox Signaling, vol. 14, no. 5, pp. 893-907, 2011.

[31] F. Chiazza, A. Couturier-Maillard, E. Benetti et al., "Targeting the NLRP3 inflammasome to reduce diet-induced metabolic abnormalities in mice," Molecular Medicine, vol. 21, no. 1, pp. 1025-1037, 2015.

[32] Y. Chen, J. Zhao, J. Du, G. Xu, C. Tang, and B. Geng, "Hydrogen sulfide regulates cardiac sarcoplasmic reticulum $\mathrm{Ca}^{2+}$ uptake via $\mathrm{K}_{\text {АтP }}$ channel and PI3K/Akt pathway," Life Sciences, vol. 91, no. 7-8, pp. 271-278, 2012.

[33] M. Najafi, S. Farajnia, M. Mohammadi et al., "Inhibition of mitochondrial permeability transition pore restores the cardioprotection by postconditioning in diabetic hearts," Journal of Diabetes \& Metabolic Disorders, vol. 13, no. 1, article 106, 2014.

[34] Y. Hu, L. I. Li, W. Yin, L. Shen, B. You, and H. Gao, "Protective effect of proanthocyanidins on anoxia-reoxygenation injury of myocardial cells mediated by the PI3K/Akt/GSK- $3 \beta$ pathway and mitochondrial ATP-sensitive potassium channel," Molecular Medicine Reports, vol. 10, no. 4, pp. 2051-2058, 2014.

[35] G. Ikeda, T. Matoba, Y. Nakano et al., "Nanoparticle-mediated targeting of cyclosporine a enhances cardioprotection against ischemia-reperfusion injury through inhibition of mitochondrial permeability transition pore opening," Scientific Reports, vol. 6, Article ID 20467, 2016.

[36] A. Kapoor, M. Collino, S. Castiglia, R. Fantozzi, and C. Thiemermann, "Activation of peroxisome proliferator-activated receptor- $\beta / \delta$ attenuates myocardial ischemia/reperfusion injury in the rat," Shock, vol. 34, no. 2, pp. 117-124, 2010.

[37] M. Zhang, C. Wang, J. Hu et al., "Notch3/Akt signaling contributes to OSM-induced protection against cardiac ischemia/reperfusion injury," Apoptosis, vol. 20, no. 9, pp. 1150$1163,2015$.

[38] D. Sun, S. Li, H. Wu et al., "Oncostatin M (OSM) protects against cardiac ischaemia/reperfusion injury in diabetic mice by regulating apoptosis, mitochondrial biogenesis and insulin sensitivity," Journal of Cellular and Molecular Medicine, vol. 19, no. 6, pp. 1296-1307, 2015.

[39] M. Collino, M. Aragno, S. Castiglia et al., "Insulin reduces cerebral ischemia/reperfusion injury in the hippocampus of diabetic rats: a role for glycogen synthase kinase-3beta," Diabetes, vol. 58, no. 1, pp. 235-242, 2009.

[40] E. R. Gross, A. K. Hsu, and G. J. Gross, "Delayed cardioprotection afforded by the glycogen synthase kinase 3 inhibitor SB216763 occurs via a $\mathrm{K}_{\mathrm{ATP}}$ - and MPTP-dependent mechanism at reperfusion," American Journal of Physiology-Heart and Circulatory Physiology, vol. 294, no. 3, pp. H1497-H1500, 2008.

[41] S. Toldo, A. Das, E. Mezzaroma et al., "Induction of microRNA21 with exogenous hydrogen sulfide attenuates myocardial ischemic and inflammatory injury in mice," Circulation: Cardiovascular Genetics, vol. 7, no. 3, pp. 311-320, 2014.

[42] A. Saxena, W. Chen, Y. Su et al., "IL-1 induces proinflammatory leukocyte infiltration and regulates fibroblast phenotype in the infarcted myocardium," The Journal of Immunology, vol. 191, no. 9, pp. 4838-4848, 2013. 


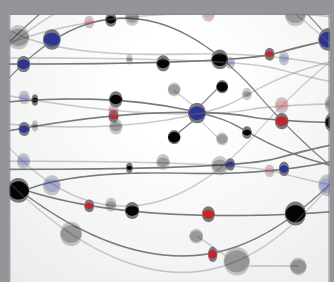

The Scientific World Journal
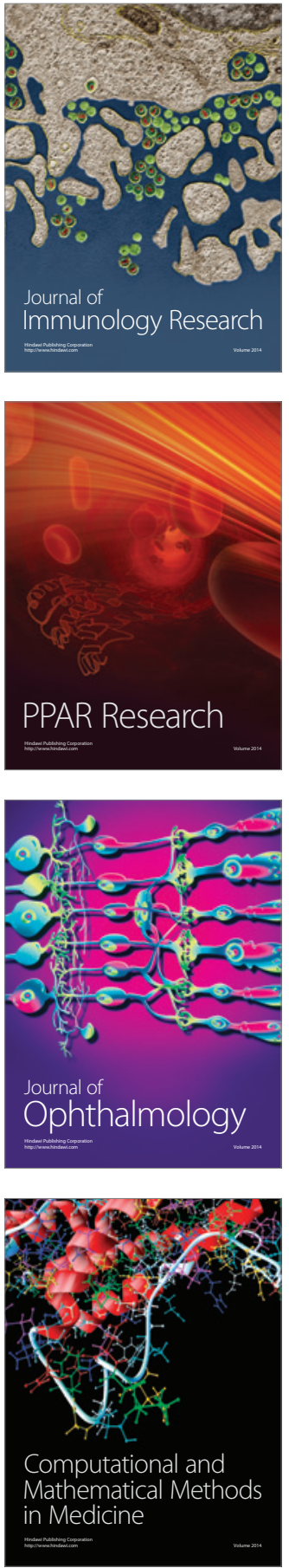

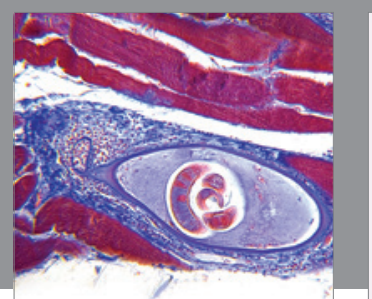

Gastroenterology Research and Practice

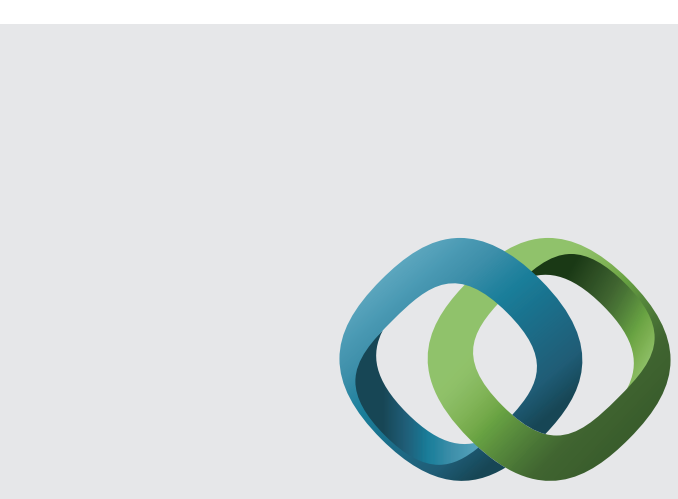

\section{Hindawi}

Submit your manuscripts at

http://www.hindawi.com
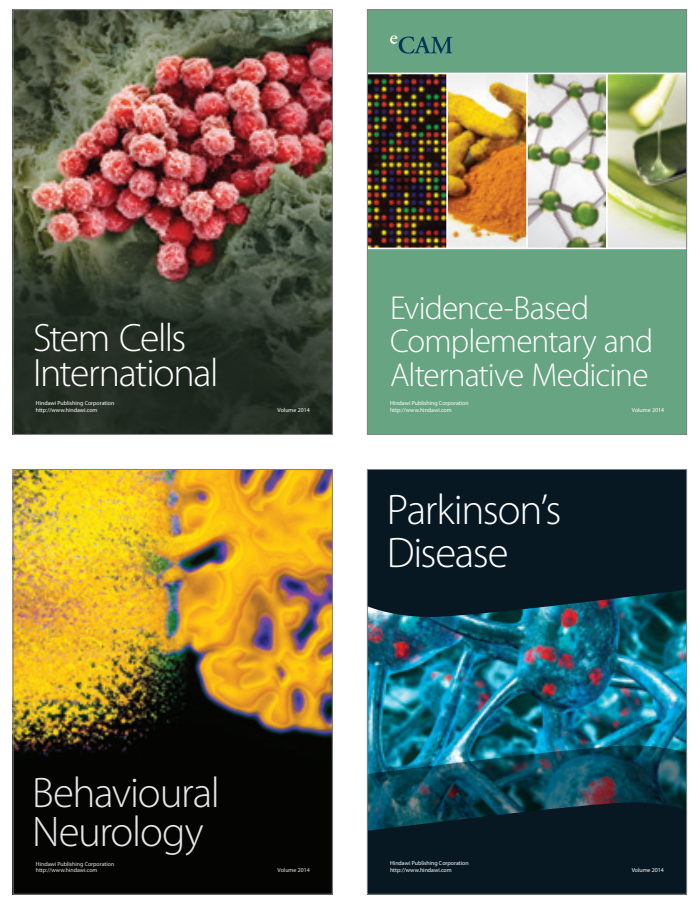
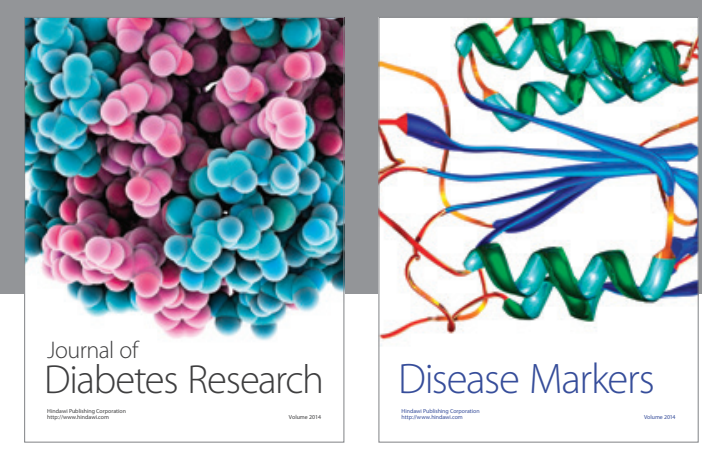

Disease Markers
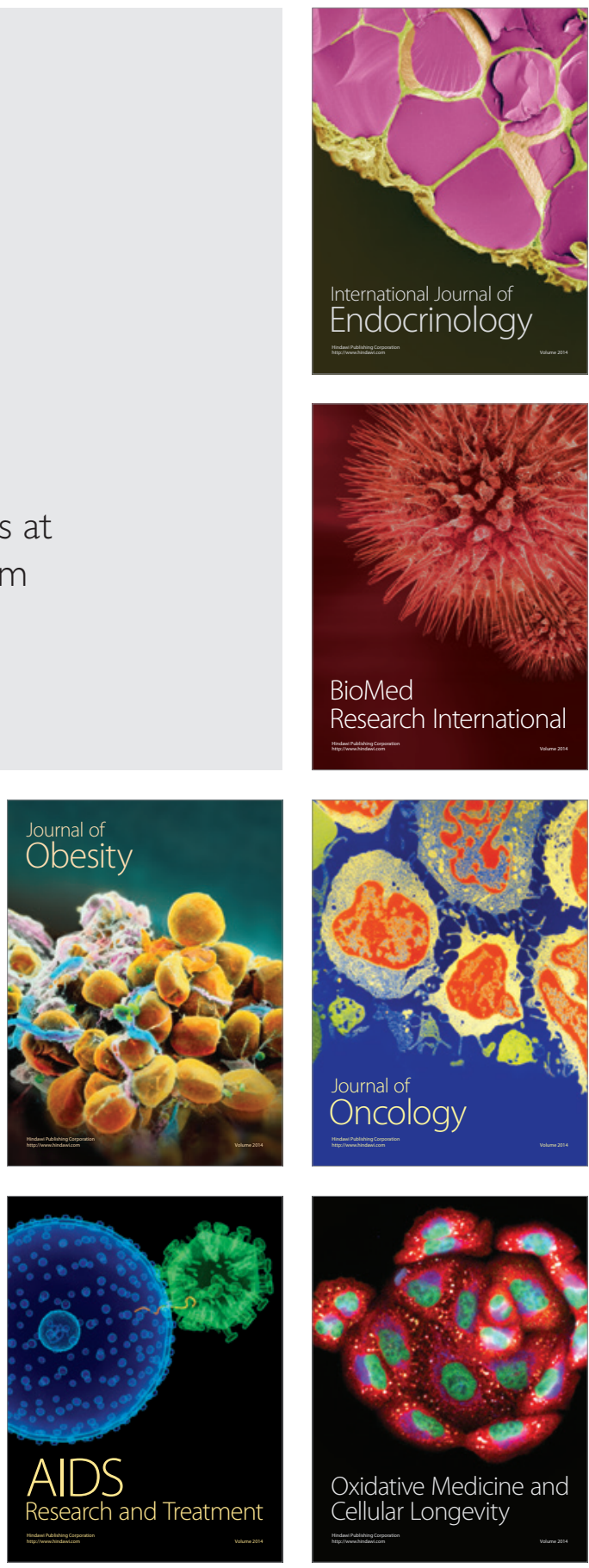